\title{
Multi-Sense Embeddings per Word
}

\author{
Masashi Sugiyama
}

\begin{abstract}
Recently, word embeddings have been used in many natural language processing problems successfully and how to train a robust and accurate word embedding system efficiently is a popular research area. Since many, if not all, words have more than one sense, it is necessary to learn vectors for all senses of word separately. Therefore, in this project, we have explored two multisense word embedding models, including Multi-Sense Skip-gram (MSSG) model and Non-parametric Multi-sense Skip Gram model (NP-MSSG). Furthermore, we propose an extension of the Multi-Sense Skip-gram model called Incremental Multi-Sense Skipgram (IMSSG) model which could learn the vectors of all senses per word incrementally. We evaluate all the systems on word similarity task and show that IMSSG is better than the other models.
\end{abstract}

\section{Introduction}

Distributed word representations, which represent words by dense, real-valued vector embeddings, have achieved remarkable performance on several natural language processing tasks (Mnih and Hinton, 2007; Collobert and Weston, 2008; Turian et al., 2010). By placing near each other words having similar semantic and syntactic roles, distributed word representations help address the curse of dimensionality and improve generalization.

Trained on large volumes of data, embeddings can obtain a substantial benefit. Compared to Brown clusters (Brown et al., 1992), distributed word representations have the advantages of substantially better scalability in the training and intriguing potential for the continuous and multi-dimensional interrelations. Thus, distributed word representations have been common input features for many tasks, such as named entity extraction (Miller et al., 2004; Ratinov and Roth, 2009) and parsing (Täckström et al., 2012). Passos et al. (Passos et al., 2014) train a Skip-gram model that injects supervision with lexicons and apply the obtained continuous vector embeddings to entity extraction. Bansal et al. (Bansal et al., 2014) adopt the Skip-gram embeddings to dependency parsing.

Despite the recent advances of leveraging distributed word representations, the polysemy and homonym are ignored in these representations in which each word type has only one vector representation. For example, the word plant has different contextual semantics relating to biology, placement, manufacturing and power generation. While the distributed representation of plant only has an embedding that is approximately the average of these contextual semantics. While in moderately highdimensional spaces a vector can be relatively 
"close" to multiple regions at a time, but this does not negate the unfortunate influence of the triangle inequality here: words that are not synonyms but are synonymous with different senses of the same word will be pulled together. How to fit the constraints of legitimate continuous gradations of semantics without the additional encumbrance of the illegitimate triangle inequalities still remains as an open research problem.

Some work (Huang et al., 2012; Reisinger and Mooney, 2010) try to discover embeddings for multiple sense per word type via preclustering the contexts of a word into discriminated senses. According to these clusters, the tokens in the corpus could be re-labelled according to different senses and then learn embeddings for these re-labeled words. However, this method loses the opportunity to jointly learn the sense vectors and the clustering.

In order to tackle this problem, a multi-sense skip gram model (MSSG) is proposed in Neelakantan et al. (2015). In this method, sense vectors and context clusters are learned jointly with the assignment of token contexts to sense. In MSSG model, the number of sense per word type is fixed but in reality, some words may have much more senses than others. Therefore, it is necessary to make the number of sense per word type flexible.

In this project, we have tried two models to solve this problem and compare their performance on word similarity task.

- Non-parametric Multi-sense Skip Gram model (NP-MSSG) (Neelakantan et al., 2015). In this model, the number of clusters (sense) is flexible and a new cluster (sense) will be added according to distance of its context to the nearest cluster (sense).

- Incremental Multi-sense Skip Gram model (IMSSG). In this model, we adapt the original MSSG, which could increase the number of sense of a word incremen- tally and stop increasing the number of sense of a word if there exists two very similar clusters (sense) already.

\section{Related Work}

Bengio et al. (2012) propose to extend the traditional idea of $n$-gram language models by replacing the conditional probability table with a neural network, representing each word token by small vector instead of an indicator variable and estimating the parameters of the neural network and these vectors jointly. To reduce the expensive computation in Bengio et al.'s work (2012), Collobert and Weston (2008) replace the max-likelihood character of the model with a max-margin approach where the network is encouraged to score the correct $n$ grams higher than randomly chosen incorrect $n$-grams. Mikolov et al. (2013a) and Mikolov et al. (2013b) propose extremely computationally efficient log-linear neural language models by removing the hidden layers of the neural networks and training from larger context windows with very aggressive subsampling. How to utilize various senses of words is also valuable for machine translation (Kong et al., 2019c,b), speech (Kong et al., 2015, 2016b,a, 2017) and dialogue (Kong et al., 2019a).

Despite the maturity of the research on learning vector representations of words, there is relatively less prior work on learning multiple vector representations for the same word type. Reisinger and Mooney (Reisinger and Mooney, 2010) propose to construct multiple sparse, high-dimensional vector representations of words. Huang et al. (Huang et al., 2012) extend this approach incorporating global document context to learn multiple dense, low-dimensional embeddings by using a recursive neural network. Neelakantan et al. (Neelakantan et al., 2015) propose two methods Multiple-sense Skip-gram (MSSG) and its non-parametric counterpart as $N P-M S S G$. These two methods are closely related to our work. In $M S S G$ model, When on- 


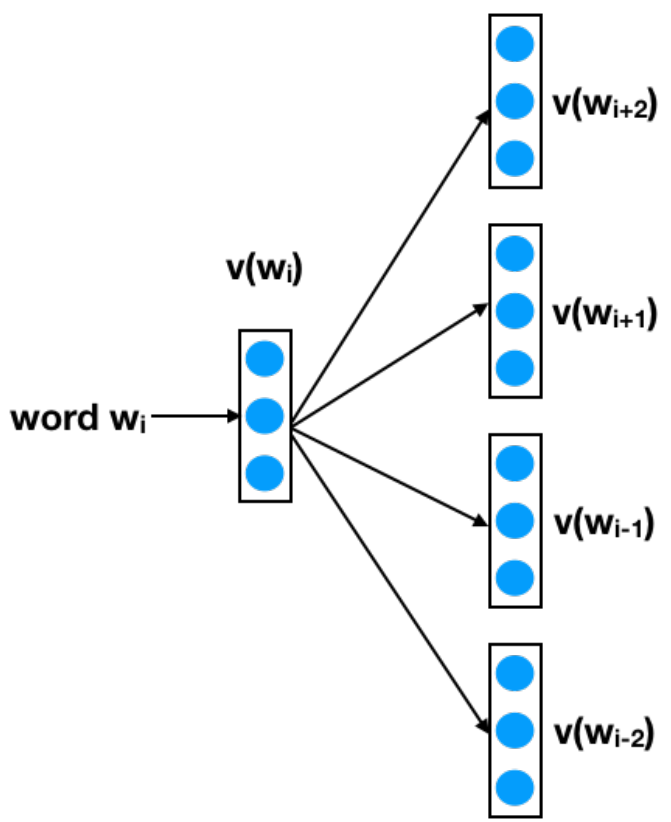

Figure 1: Architecture of the Skip-gram model with window size $R=2$. Context $c_{i}$ of word $w_{i}$ is $w_{i-2}, w_{i-1}, w_{i+1}, w_{i+2}$

line training with a particular token, Neelakan$\tan$ et al.adopt the average of its context words' vectors to select the token's sense that is closest and update the gradient on the corresponding sense. While in NP-MSSG model, Neelakantan et al.build on facility location (Meyerson, 2001): a new cluster is created with probability proportional to the distance from the context to the nearest sense.

\section{Skip-gram model}

The Skip-gram model learns word embeddings though predicting the surrounding words in a sentence. In the Skip-gram, $v(w) \in R^{d}$ is the embedding vector for the word $w$ where $d$ is the dimension of the embedding. In more details, given a pair of words $\left(w_{i}, w_{j}\right)$, the probability that the word $w_{j}$ is observed in the context of word $w_{i}$ is given by:

$$
P\left(D=1 \mid v\left(w_{i}\right), v\left(w_{j}\right)\right)=\frac{1}{e^{-v\left(w_{j}\right)^{T} v\left(w_{i}\right)}}
$$

Also, the probability of not observing word $w_{j}$ in the context of word $w_{i}$ is $1-P(D=$ $\left.1 \mid v\left(w_{i}\right), v\left(w_{j}\right)\right)$. Therefore, given a training set including the sequence of words $w_{1}, \ldots, w_{T}$, the word embeddings are learned by maximizing the following objective:

$$
\begin{aligned}
J= & \sum_{\left(w_{j}\right) \in c_{i}^{+}} \log P\left(D=1 \mid v\left(w_{i}\right), v\left(w_{j}\right)\right) \\
& +\sum_{\left(w_{j}^{\prime}\right) \in c_{i}^{-}} \log P\left(D=0 \mid v\left(w_{i}\right), v\left(w_{j}^{\prime}\right)\right)
\end{aligned}
$$

where $w_{i}$ is the $i$-th word in the training set, $c_{i}^{+}$is the set of context words of word $w_{i}$ and $c_{i}^{-}$is the set of randomly selected context words for the word $w_{i}$. In more details, the set of context words for a given word $w_{i}$ is $c_{i}^{+}=\left\{w_{i-R}, \ldots, w_{i-1}, w_{i+1}, w_{i+R}\right\}$, where $R$ is window size. A sample of the Skip-gram model is shown in Fig. 1

\section{Methodology}

\subsection{Multi-Sense Skip-gram (MSSG) model}

In the MSSG model, each word $w$ has a global vector $v_{g}(w)$ and there is an embedding $v_{s}(w, k)$ and a context cluster $\mu(w, k)(k=$ $1, \ldots, K)$ for each sense of the word, where $K$ is a hyperparameter. Note that $K$ is universal across all words which means that the number of sense for each word is the same. The global vector $v_{g}(w)$ is the average vector of all sense vectors.

Just like the Skip-gram model, in the MSSG model, given a word $w_{i}$, we could obtain the context of this word $c_{i}=$ $\left\{w_{i-R}, \ldots, w_{i-1}, w_{i+1}, w_{i+R}\right\}$, then we could calculate the vector representation of the context $c_{i}$, i.e., $v_{\text {context }}\left(c_{i}\right)=\frac{1}{2 * R} \sum_{w_{j} \in c_{i}} v_{g}\left(w_{j}\right)$. Note that here we use the global vector instead of its sense vectors of the context words to avoid the high computational cost. After getting the context vector presentation, we could 


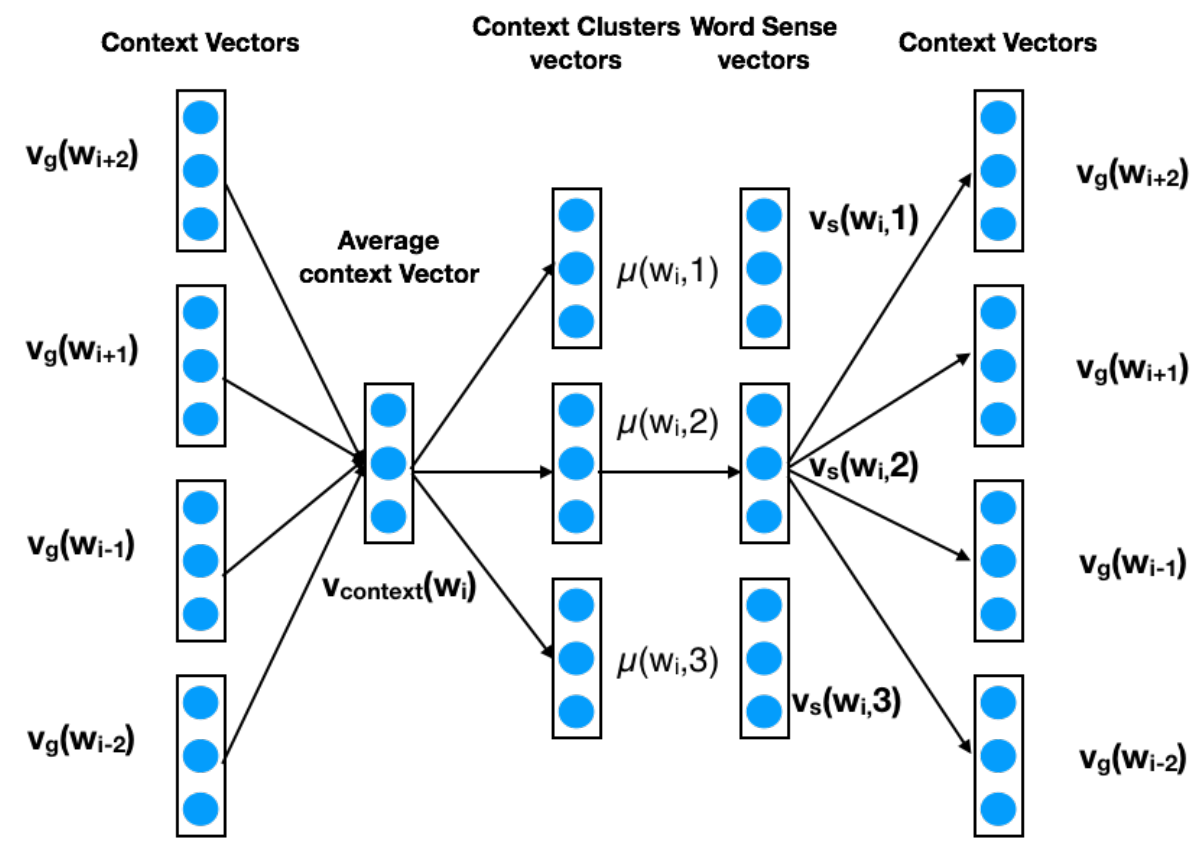

Figure 2: Architecture of the Multi-Sense Skip-gram model with window size $R=2$ and the number of sense $K=3$. Context $c_{i}$ of word $w_{i}$ is $w_{i-2}, w_{i-1}, w_{i+1}, w_{i+2}$. The sense of the word $w_{i}$ is predicted by finding the cluster vector of the context which is nearest to the average of the context vectors.

predict the sense of the word $w_{i}, s_{i}$ according to:

$s_{i}=\operatorname{argmax}_{k=(1, \ldots, K)} \operatorname{sim}\left(\mu\left(w_{i}, k\right), v_{\text {context }}\left(c_{i}\right)\right)$

Intuitively, we calculate the similarity between context vector and each cluster of word $w_{i}$ and choose the nearest one as the predicted sense of the word $w_{i}$ in this context. Here cosine similarity is used in experiments. After predicting the sense of word $w_{i}$, the context cluster vector $\mu\left(w_{i}, s i\right)$ is also updated since context $c_{i}$ is added to $s_{i}$ context cluster, we also could calculate the probability of a word $w_{j}$ observing in the context of word $w_{i}$ by:

$P\left(D=1 \mid v_{s}\left(w_{i}, s_{i}\right), v_{g}\left(w_{j}\right)\right)=\frac{1}{e^{-v_{g}\left(w_{j}\right)^{T} v_{s}\left(w_{i}, s_{i}\right)}}$

Similar to the Skip-gram model, the objec- tive function of the MSSG model is:

$$
\begin{aligned}
J= & \sum_{\left(w_{j}\right) \in c_{i}^{+}} \log P\left(D=1 \mid v_{s}\left(w_{i}, s_{i}\right), v_{g}\left(w_{j}\right)\right) \\
& +\sum_{\left(w_{j}^{\prime}\right) \in c_{i}^{-}} \log P\left(D=0 \mid v_{s}\left(w_{i}, s_{i}\right), v_{g}\left(w_{j}^{\prime}\right)\right)
\end{aligned}
$$

where $c_{i}^{+}$is the set of context words and $c_{i}^{-}$is the set of noisy words for the word $w_{i}$.

\subsection{Non-Parametric MSSG model (NP-MSSG)}

One major problem of the MSSG model is that the number of sense for each word is fixed which is $K$. However, some words may have more than $K$ senses and other words have less than $K$ senses. The NP-MSSG model is able to learn various number of sense per word. Similar to the MSSG model, each word $w$ is associated with sense vectors, contexts clusters 
and a global vector. Different from the MSSG model, the number of sense vectors and context clusters is unknown at first. The first sense vector and context cluster for each word will be created on its first occurrence in the training data. After creating the first context cluster for a word, a new context cluster and a sense vector are created online during training when the similarity between the vector representation of the context with every existing cluster center of the word is less than $\lambda$, where $\lambda$ is a hyperparameter of the model. Therefore, the major difference between NP-MSSG and MSSG is the process of sense selection. In the sense selection of the NP-MSSG model, we also have to calculate the context vector $v_{\text {context }}\left(c_{i}\right)=\frac{1}{2 * R} \sum_{w_{j} \in c_{i}} v_{g}\left(w_{j}\right)$ first. Let $k\left(w_{i}\right)$ be the number of context clusters currently associated with the word $w_{i} . s_{i}$, the sense of word $w_{i}$ is given by:

$s_{i}=\left\{\begin{array}{c}k\left(w_{i}\right)+1 \text { if } \max _{k=1, \ldots, k\left(w_{i}\right)}\{\operatorname{sim} \\ \left.\left(\mu\left(w_{i}, k\right), v_{\text {context }}\left(c_{i}\right)\right)\right\}<\lambda \\ k_{\max } \text { otherwise }\end{array}\right.$

where $\mu\left(w_{i}, k\right)$ is the cluster vector of the $k$-th sense of the word $w_{i}$ and $k_{\max }=$ $\operatorname{argmax}_{k=\left(1, \ldots, k\left(w_{i}\right)\right.} \operatorname{sim}\left(\mu\left(w_{i}, k\right), v_{\text {context }}\left(c_{i}\right)\right)$.

The cluster center is the average of the vector representations of all the contexts which belong to that cluster. If $s_{i}=k\left(w_{i}\right)+1$, a new context cluster and a new sense vector are created for the word $w_{i}$.

In summary, the NP-MSSG model and the MSSG model described previously differ only in the way that word sense discrimination is performed. The objective function and the probabilistic model associated with observing a (word, context) pair given the sense of the word remain the same.

\section{References}

Mohit Bansal, Kevin Gimpel, and Karen Livescu. 2014. Tailoring continuous word representations for dependency parsing. In $A C L$.

Peter F Brown, Peter V Desouza, Robert L Mercer, Vincent J Della Pietra, and Jenifer C Lai. 1992. Class-based n-gram models of natural language. Computational linguistics 18(4):467-479.

Ronan Collobert and Jason Weston. 2008. A unified architecture for natural language processing: Deep neural networks with multitask learning. In $I C M L$.

Eric H Huang, Richard Socher, Christopher D Manning, and Andrew Y Ng. 2012. Improving word representations via global context and multiple word prototypes. In $A C L$.

Xiang Kong, Jeung-Yoon Choi, and Stefanie Shattuck-Hufnagel. 2015. Analysis of distinctive feature matching with random error generation in a lexical access system. The Journal of the Acoustical Society of America 138(3):17801780 .

Xiang Kong, Jeung-Yoon Choi, and Stefanie Shattuck-Hufnagel. 2017. Evaluating automatic speech recognition systems in comparison with human perception results using distinctive feature measures. In 2017 IEEE International Conference on Acoustics, Speech and Signal Processing (ICASSP). IEEE, pages 5810-5814.

Xiang Kong, Preethi Jyothi, and Mark HasegawaJohnson. 2016a. Performance improvement of probabilistic transcriptions with languagespecific constraints. Procedia Computer Science $81: 30-36$.

Xiang Kong, Bohan Li, Graham Neubig, Eduard Hovy, and Yiming Yang. 2019a. An adversarial approach to high-quality, sentimentcontrolled neural dialogue generation. arXiv preprint arXiv:1901.07129.

Xiang Kong, Zhaopeng Tu, Shuming Shi, Eduard Hovy, and Tong Zhang. 2019b. Neural machine translation with adequacy-oriented learning. In Proceedings of the AAAI Conference on Artificial Intelligence. volume 33, pages 6618-6625.

Xiang Kong, Qizhe Xie, Zihang Dai, and Eduard Hovy. 2019c. Fast and simple mixture of softmaxes with bpe and hybrid-lightrnn for language 
generation. In Proceedings of the AAAI Conference on Artificial Intelligence. volume 33, pages 6626-6633.

Xiang Kong, Xuesong Yang, Mark HasegawaJohnson, Jeung-Yoon Choi, and Stefanie Shattuck-Hufnagel. 2016b. Landmark-based consonant voicing detection on multilingual corpora. arXiv preprint arXiv:1611.03533 .

Adam Meyerson. 2001. Online facility location. In Foundations of Computer Science, 2001. Proceedings. 42nd IEEE Symposium on. IEEE, pages $426-431$.

Tomas Mikolov, Kai Chen, Greg Corrado, and Jeffrey Dean. 2013a. Efficient estimation of word representations in vector space. ICLR .

Tomas Mikolov, Ilya Sutskever, Kai Chen, Greg S Corrado, and Jeff Dean. 2013b. Distributed representations of words and phrases and their compositionality. In NIPS.

Scott Miller, Jethran Guinness, and Alex Zamanian. 2004. Name tagging with word clusters and discriminative training. In $H L T-N A A C L$.

Andriy Mnih and Geoffrey Hinton. 2007. Three new graphical models for statistical language modelling. In ICML.

Arvind Neelakantan, Jeevan Shankar, Alexandre Passos, and Andrew McCallum. 2015. Efficient non-parametric estimation of multiple embeddings per word in vector space. In EMNLP.

Alexandre Passos, Vineet Kumar, and Andrew McCallum. 2014. Lexicon infused phrase embeddings for named entity resolution. CoNLL .

Lev Ratinov and Dan Roth. 2009. Design challenges and misconceptions in named entity recognition. In Proceedings of the Thirteenth Conference on Computational Natural Language Learning. ACL.

Joseph Reisinger and Raymond J Mooney. 2010. Multi-prototype vector-space models of word meaning. In $H L-A C L$.

Oscar Täckström, Ryan McDonald, and Jakob Uszkoreit. 2012. Cross-lingual word clusters for direct transfer of linguistic structure. In Proceedings of the 2012 conference of the North American chapter of the association for computational linguistics: Human language technologies. ACL.
Joseph Turian, Lev Ratinov, and Yoshua Bengio. 2010. Word representations: a simple and general method for semi-supervised learning. In $A C L$. 